PHILOSOPHICAL TOPICS

VOL. 39, NO. 1, SPRING 2011

\title{
Embodied Social Cognition
}

Shannon Spaulding

Oklahoma State University

\begin{abstract}
In this paper I evaluate embodied social cognition, embodied cognition's account of how we understand others. I identify and evaluate three claims that motivate embodied social cognition. These claims are not specific to social cognition; they are general hypotheses about cognition. As such, they may be used in more general arguments for embodied cognition. I argue that we have good reasons to reject these claims. Thus, the case for embodied social cognition fails. Moreover, to the extent that general arguments for embodied cognition rest on these premises, they are correspondingly uncompelling.
\end{abstract}

\section{INTRODUCTION TO EMBODIED SOCIAL COGNITION}

The embodied cognition (EC) account of social cognition, propounded most notably by Shaun Gallagher $(2005,2008)$ and Daniel Hutto $(2008)$, holds that basic, nonmentalistic, embodied practices are developmentally fundamental and constitute the primary way we understand others even as adults. These embodied practices are constituted by 'primary intersubjectivity' and 'secondary intersubjectivity'. Primary intersubjectivity involves the capacity to see emotions and basic intentions in facial expressions, voice intonations, gestures, and bodily postures. A capacity for primary intersubjectivity begins very early in life. For instance, infants are able to 
discriminate faces from non-faces, determine which things in their environment are persons, detect goal-directed intentional movement, and detect emotions (Gallagher 2005, 204).

After the first year, infants start to develop the capacity for secondary intersubjectivity, which unites primary intersubjectivity with the capacity for joint attention. The infant moves from basic one-on-one interactions to contexts of shared attention. In addition to tracking eye movement, detecting intentional behavior and emotions, with the development of secondary intersubjectivity, the child develops the capacity to engage in shared attention behaviors. At this stage, an object or event can become a focus among people. The child learns to follow gazes, point, and communicate with others about objects of shared attention (Gallagher 2005, 207). This combination of the mechanisms of primary intersubjectivity and the capacity for joint attention allows for a more sophisticated understanding of the social world surrounding the child, but still this understanding is based solely on basic, nonmentalistic, embodied practices (de Bruin 2008; Gallagher 2008; Gallagher and Hutto 2008; Hutto 2008).

EC theorists claim that the intersubjective practices of everyday human life acquire meaning through primary and secondary intersubjectivity. We come to understand others' body language, eye movements, gesturing, and facial expressions through engaging in primary and secondary intersubjectivity. On the basis of primary and secondary intersubjectivity, children are able to interact socially before they learn about mental states much later in development. The capacities that allow them to interact socially are necessary to develop the capacity to think about others' thoughts. ${ }^{1}$ Thus, the embodied practices are developmentally fundamental. Furthermore, these intersubjective practices constitute the predominant way of understanding others even into adulthood. In ordinary interactions, we do not have to postulate beliefs, desires, or other hidden mental states to make sense of others' actions because we have a direct perceptual understanding of their intentions, emotions, and feelings that makes mindreading - attributing mental states in order to explain and predict behavior-superfluous. ${ }^{2}$

EC's direct social perception is a close kin to Alva Noë's enactive perception, both of which stem ultimately from J. J. Gibson's ecological psychology. All of these accounts eschew the representationalism of standard cognitive science, arguing that the information in the environment is both necessary and sufficient for the various kinds of perception, where the "pick up" of this information does not depend on representational states. For example, Noë, following Gibson, argues that visual perception does not consist in mentally constructing an intermediary representation of a visual scene. Rather, all of the information necessary for perception is out there in the environment, and perception consists in actions that pick up that information, bypassing the need for representational intermediaries (Gibson 1986; Noë 2006). Direct perception is a noninferential awareness of things in the external world. Similarly, direct social perception is a noninferential awareness of others' emotions and intentions. Gallagher and Hutto argue that social cognition does not 
consist in representing others' mental states and explaining and predicting others' behavior on the basis of attributions of mental states. Embodied intersubjective practices are necessary and usually sufficient for social understanding. People's behaviors usually express their emotions, intentions, and feelings, so there is no need to for us to go beyond embodied cues to understand others. Instead, in perceiving others' behavior, we directly perceive their intentions, emotions, and feelings. Direct social perception is the foundation of embodied accounts of social cognition.

\section{THE ARGUMENT FOR EMBODIED SOCIAL COGNITION}

In this section, I shall lay out the argument for embodied social cognition. The argument rests on three claims, which are meant to undermine the idea that mindreading is our primary and pervasive way of understanding others. The argument is as follows:

1. Only a sentential medium could support contentful, representational thought.

2. Natural language is the only legitimate source for a sentential medium of thought. This implies that pre- and non-linguistic creatures cannot have contentful, representational thought.

3. The best explanation of pre- and non-linguistic cognition is in terms of nonmentalistic intentional attitudes.

This argument, if successful, would undermine the idea that mindreading is a fundamental and important aspect of social cognition. For mindreading requires having and attributing contentful, representational thought. If we are not capable even of having contentful, representational thought until we master natural language, around age three, then it is very unlikely that the social cognitive skills children display are grounded in mindreading. If correct, this would bolster EC's claim that mindreading is a late-developing, specialized, rarely used skill.

\subsection{SENTENTIALISM}

EC theorists hold that our ordinary intersubjective practices are nonmentalistic, and as such do not involve mindreading. The argument for this claim begins with an examination of the basic requirements for mindreading. Mindreading involves attributing a mental state to another in order to explain and predict behavior. The mindreader has a mental state about a target's mental state. A minimum constraint on the mental states constituting mindreading is that they be contentful, representational states. That is, in order for a mental state $(\mathrm{M})$ to be about another mental state $\left(\mathrm{M}^{\star}\right), \mathrm{M}$ must represent $\mathrm{M}^{\star}$ under a certain description or mode of presentation. There is an enormous literature on the related topics of mental content, sense 
and reference, intentionality, and representation. At this point we need not delve deeply into the details of these debates. The important point here is that a mindreader must be capable of having contentful, representational thoughts.

Mindreaders must be capable of having thoughts with mental content. So, what are the requirements for thoughts with mental content? Hutto provides a list of pretty typical requirements: thoughts with mental content must be intentional, intrinsically represent that which they are about, and be part of a structured system that allows for recombination, creativity, and performance of logical operations (Hutto 2008, 46, 78-81). Propositional attitudes are a paradigm case of the kind of mental state that satisfies these requirements.

In fact, Hutto argues that only propositional attitudes satisfy these requirements. Only a sententially structured medium could support contentful, representational thought. Jerry Fodor's (1975) case for the Language of Thought (LOT) is one influential argument for the sententialism. The LOT hypothesis holds that our thinking is conducted in mentalese, an innate, sententially structured system of thought. Hutto agrees with much of Fodor's reasoning about propositional attitudes. Hutto agrees that propositional attitudes involve mental representations that belong to a representational, symbolic system that is characterized by a combinatorial syntax and semantics. He says, "For a creature to have an attitude directed at a proposition (and not just a worldly offering) - for it to apprehend a state of affairs intensionally, so to speak - it must have the capacity to direct its attention at that state of affairs via structured vehicles of thought of some appropriate sort" (Hutto 2008,87 ). Hutto agrees that only a sentential medium can explain the productivity, systematicity, compositionality, and truth-preserving inferential capacity of propositional thought. He says, "Only sentences have the right syntactic and semantic properties for expressing propositional contents” (Hutto 2008, 87). His disagreement is with the claim that this kind of thought is available to preverbal and nonverbal creatures.

\subsection{THE NECESSITY OF NATURAL LANGUAGE}

Hutto argues that only natural language users are capable of contentful, representational thought. ${ }^{3}$ Arguing against the central element of Fodor's LOT, Hutto claims that the analogy between public language and mentalese breaks down when it comes to how mentalese sentences get their meaning. In public language, this is unmysterious: the meanings of linguistic signs are derived from public conventions. Mentalese, however, is meant to have nonderived content. That is, the meaning of mentalese symbols is not derived from other symbols or conventions. Moreover, according to LOT, public language sentences acquire their semantic properties from mentalese, which makes even more pressing the need for an account of how mentalese symbols acquire their meaning.

Hutto argues that there is no theory of content that could explain how mentalese symbols come to be meaningful. LOT theorists typically rely on naturalistic 
theories of content determination. Such theories attempt to ground the content of a mental representation (e.g., a mentalese symbol) in the information it carries about what causes the mental representation to be tokened. Causal-informational relations are necessary for determining the content of a representation, but not sufficient. Fred Dretske, for example, argues that in addition to causal-informational relations, a representation must have the selected (via learning or evolution) function of carrying information about what causes the representation (1988). ${ }^{4}$ This accommodates the fact that whereas causal-informational relations are ubiquitous, representations are relatively rare. It also promises to explain how representations might misrepresent (a crucial feature of any account of representation).

According to Hutto, Dretkse's account and other naturalistic accounts like it are misguided. Hutto disagrees with both the relevance of covariation and the requirements for representation. First, he claims, things do not carry information about other things. If covariation implied a natural relation of indication, "the world would be everywhere silently referring or marking truths about itself (a strange metaphysics indeed)" (Hutto 2008, 49). Naturalistic accounts of content fall prey to the containment metaphor; i.e., the idea that things in the world carry or contain information about how things stand in the world. The containment metaphor leads theorists to believe that signals and signs must be decoded and understood by organisms in order for such signals and signs to successfully guide action. This, however, is wrong. All that successful responding requires is the existence of the correlation between a sign or signal and a distal state of affairs. Organisms need not represent or in anyway cognitively register the correlation. ${ }^{5}$

Second, Hutto asserts that signs and signals only represent something if they are used as entities that stand for something else. He does not elaborate his reasoning here, but presumably the idea is that explaining how LOT symbols get their content is difficult_-perhaps impossible-on the proper account of representation. Mentalese symbols would have to somehow be used as entities that stand in for what they represent. We can see how this would work for thinking in natural language. Mental representations stand in for natural language words and sentences. However, it is unclear how we can tell a similar story for mentalese.

On the EC view, one cannot even engage in propositional thought until one becomes a natural language user. Thus one cannot be even a candidate mindreader until one has mastered natural language. In support of the idea that natural language enables propositional thought, Hutto cites empirical evidence regarding socalled oral deaf children, congentially deaf children who are reared in nonsigning households. Oral deaf children perform very poorly on mindreading tasks. They seem not to understand the concept of BELIEF. It is not until these children learn sign language that they begin to pass mindreading tasks, such as the false-belief task (Peterson and Siegal 2000). These data allegedly support the idea that facility with natural language is necessary for propositional thought. Hutto also takes as evidence for his account the facts that normally developing children's performance on standard false-belief tasks improves at roughly the same time that children's linguistic 
abilities begin to mature, and that mastery of language improves performance on mindreading tasks $(2008,131-37)$. These mindreading tasks require the subjects to reason about others' mental states. Though Hutto does not regard reasoning about others' mental states as an ordinary part of our social interactions, he does think performance on these tasks is informative. Reasoning about mental states requires contentful, representational thought. These data suggest that natural language confers the ability to reason about mental states. Hutto takes this as evidence for his view that having and attributing propositional attitudes are dependent on natural language.

\subsection{NONMENTALISTIC INTENTIONAL ATTITUDES}

EC theorists distinguish between having and attributing propositional attitudes and having and attributing intentional attitudes. Hutto argues that only the former involves contentful mental representation (Hutto 2008, 45). That is, only propositional attitudes satisfy the four requirements listed above. Propositional attitudes are contentful representations with syntactic and semantic properties. Having a propositional attitude involves standing in a certain relation to a proposition or thought. For a subject to attribute a propositional attitude to a target the subject must be able to represent the target as standing in a certain relation to a particular proposition or thought.

In contrast, according to EC, intentional attitudes are noncontentful, nonrepresentational states. Intentional attitudes, they argue, are simply perceptual sensitivities and appropriate responsiveness to specific environmental particulars. The combination of informational sensitivities and appropriate responsiveness enables the coordination of successful actions in particular environments. On this view, having an intentional attitude consists in being sensitive and responsive to features of the environment, and attributing an intentional attitude consists in detecting others' sensitivities to specific environmental particulars. This does not entail representing the target's mental states, the target's informational sensitivities, or things in world. No representations are involved in having and attributing intentional attitudes. On this account, intention understanding does not satisfy any of the criteria for thoughts with mental content that I listed above. This entails that intention understanding does not involve contentful mental representations, a basic requirement of mindreading (de Bruin 2008).

Here is a general sketch of how this account of intention is supposed to work. Intentionality and affect are expressed by the way organisms carry themselves. Our basic intentions and emotions manifest natural signs. These natural signs, which are basically the same for everyone, were selected for and shaped by natural selection because they benefited our ancestors in communicating and coordinating actions. "[I]nfants and adults are directly responsive to the psychological situation of others because they are informationally sensitive to a special class of natural sign-the expressions of intentional and affective attitudes, as revealed in another's 
gaze, gesture, facial comportment, and so on" (Hutto 2008, 117). Understanding others' intentions and emotions consists in sensitivity and appropriate responsiveness to these natural signs. Importantly, successful intention understanding does not require representing or inferring the meaning of these natural signs. ${ }^{6}$ We do not need to represent these natural signs as signs for them to shape our social understanding and interactions. If all this is correct, then intentional attitudes do not involve contentful mental representations.

This distinction between nonmentalistic (i.e., noncontentful and nonrepresentational) intentional attitudes and propositional attitudes is a departure from the standard cognitivist account. EC theorists argue that we have good reasons to adopt this alternative account. Nonverbal animals and preverbal children engage in some sorts of social cognitive activity. In particular, they engage in primary and secondary intersubjectivity; e.g., recognition and response to agency, emotions, and goals. According to EC, however, having and attributing propositional attitudes depends on facility with natural language. This implies that these basic social capacities must be grounded in having and attributing intentional attitudes, not propositional attitudes because these organisms do not have natural language. Moreover, since having and attributing propositional attitudes requires natural language and since basic social capacities are in play before natural language develops, our basic social capacities do not involve having and attributing propositional attitudes; i.e., mindreading (Gallagher 2008; Hutto 2008).

The final element in the argument for EC's account of social cognition holds that the nonmentalistic account of intentional attitudes better explains nonverbal and preverbal behavior than accounts that ascribe propositional thought to nonverbal and preverbal organisms. EC theorists claim that we can explain all nonverbal behavior without invoking contentful mental representations. Hutto says, "It is my contention that when it comes to understanding our primary modes of engagement, content-based accounts are in any case utterly surplus to requirements" $(2008,49)$.

Hutto considers several examples of nonverbal behavior that theorists often take to imply contentful, representational thought; e.g., the honeybee dance. When foraging honeybees find a useful item (e.g., nectar, pollen, or a new hive site), they fly back to the hive and perform a waggle dance. The waggle dance has two variables: the length of the dance and the angle of the dance relative to the sun and the hive. Suppose the foraging honeybee has found a source of nectar and returns to the nest to perform a waggle dance. The length of the bee's dance closely corresponds to the distance from the hive to the nectar, and the angle of the dance, relative to the Earth's surface, closely corresponds to the direction to the nectar, relative to the current solar azimuth (the compass direction of the sun). The honeybee waggle dance is performed in the hive where it is dark and cues from the sun are absent. The bees adjust the angle of their waggle dance for a particular source of nectar as it gets later in the day, and their dance accurately indicates the location of the nectar when performed at night and even many hours or days after the discovery of the nectar (Dyer 2002; Riley et al. 2005; Von Frisch 1967). 
Many theorists argue that the honeybee dance indicates that honeybees have representational thought. They argue that the foraging bees represent the location of the nectar and communicate that location to other bees. The bees in the hive decode the communication in order to represent the location of the nectar (Carruthers 2006; Millikan 2005). The content of the bees' representation is the location of the nectar.

Hutto argues that honeybees' behavior does not imply that they have representational, contentful thought. The bee that has returned from foraging is simply genetically programmed to dance in response to finding nectar. The observing bees do not use the foraging bee's waggle dance to represent, indicate, or stand for anything else- - a requirement on Hutto's account of representation. The foraging bee's behavior simply directly guides the other bees' behavior. In Hutto's terminology, it is a local indexical guide, which has the proper function of generating a sequence of behavior with respect to specific worldly offerings (Hutto 2008, 54-56). This proper function is an evolved pattern of behavior that was selected for due to its evolutionary advantageousness. Bees that engaged in this behavior lived longer and produced more offspring than those that did not. For this sort of behavior to be successful, the honeybees must be informationally sensitive and responsive to the relations between the sun, hive, and nectar. But the bees need not represent these relations. The waggle dance directly guides action. In general, such natural signs directly guide action without the mediation of any contentful representations.

Of course, we could describe the bee behavior in terms of contentful representations - we could attribute beliefs and desires to the bees and, on that basis, predict their behavior-but Hutto regards this as an unhelpful fiction. He thinks this way of talking does not illuminate the issue, and it relies on the dubitable notion that there is a theory of content that could substantiate such attributions. The general lesson is that nonverbal organisms' successful responding depends only on the existence of a correlation between specific natural signs and a distal state of affairs, and a capacity to act appropriately in response to the natural signs (Hutto 2008, 47). Organisms do not decode the natural signs to which they are sensitive or represent this correlation in order to act successfully. Preverbal and nonverbal organisms' interactions are not driven by propositional thought. Instead, these interactions are driven by intentional attitudes, which are noncontentful and nonrepresentational. Hence, preverbal and nonverbal organisms' interactions cannot be precursors to mindreading because they do not involve contentful, representational thought.

\subsection{ARGUMENT SUMMARY}

To summarize the argument, EC theorists argue that preverbal and nonverbal organisms have a capacity for social cognition, but this cannot consist in attributing mental states; i.e., mindreading. Mindreading requires that the mindreader be able to have contentful, representational mental states about a target's mental states. However, (1) only a sententialist medium could support contentful, representa- 
tional mental states. (2) LOT-the main sententialist account of nonverbal propositional thought - is hopelessly problematic. Thus, preverbal and nonverbal organisms' cognition cannot consist in contentful, representational mental states, and so their social cognition cannot consist in mindreading. Finally, (3) preverbal and nonverbal organisms' behavior is best explained by appeal to basic, nonmentalistic embodied practices. Notice that although these claims are employed in service of an argument for embodied social cognition, they are not specific to social cognition. These premises may be used in a more general argument for embodied cognition. In the next sections, I shall challenge claims (1), (2), and (3). ${ }^{7,8}$ Arguing against these three claims undermines the case for embodied social cognition.

\section{CRITIQUE OF EC}

The goal of this paper is to show that the EC account of social cognition is not viable. This involves rejecting some of EC's foundational claims. Of particular importance are claims (1)-(3) described above. Section 3 challenges these claims. My aim in section 3.1 is to dismiss EC's argument that nonverbal intersubjective practices could not possibly involve mindreading. I shall argue that nonverbal organisms can satisfy Hutto's conditions for having contentful, representational mental states, and so at least are candidate mindreaders. In section 3.2 I shall argue that even if sententialism were true, the LOT hypothesis is not defeated by EC's arguments. In section 3.3 I shall argue that the flexibility of nonverbal behavior counts against EC's claim that nonverbal organisms' behaviors are nonmentalistic. Finally, in section 3.4 I shall dismiss the evidence Hutto corrals for his account. Together, these claims undermine the case for EC's account of social cognition.

\subsection{AGAINST SENTENTIALISM}

Hutto argues that only a sentential medium could support contentful mental representations and that only organisms possessing natural language are capable of mindreading. From various passages in his book, we can glean several individually necessary and (I presume) jointly sufficient conditions on genuine contentful, representational thought. Contentful, representational mental states must:

1. be intentional,

2. be a part of a structured system that allows for recombination and creativity,

3. be a part of a system over which logical operations can be performed (including conjunction, negation, disjunction, and quantification), and

4. intrinsically represent, rather than just resemble, what they are about (2008, 46, 78-81).

I shall argue that thinking in images, mental maps, and models ${ }^{9}$ satisfies these four criteria when the criteria are understood in the right way. Thinking in these mediums 
can satisfy Hutto's necessary and jointly sufficient conditions for contentful, representational thought. This is meant to undermine the claim that nonverbal organisms cannot mindread because they are incapable of having contentful, representational thought.

I shall explain how images, mental maps, and models, what I will call iconic representations, satisfy each of these criteria, but first a few caveats. In what follows, I do not claim that all kinds of thinking take place in iconic representations. Recall that the topic of dispute is nonverbal thought. My claim is that there is a medium that is not dependent on natural language that could support genuine thought. I am not claiming that all genuine thought must be or is grounded in iconic representations.

The second caveat is about the sophistication of thought grounded in iconic representations. Hutto argues that iconic representations could not support the full range of truth-preserving logical inferences characteristic of genuine thought, and so they cannot be the vehicles of genuinely contentful mental representations $(2008,107,232)$. I reject this idea. Of course, for nonverbal creatures to be capable of genuine contentful mental representation they must be capable of thinking in a representational system of some sort, but it need not be as semantically and logically sophisticated as the linguistically mediated thought in which adult humans engage. Iconic representations may not have the expressive power or logical and semantic complexity of linguistically mediated thought. But that is perfectly consistent with the idea that nonverbal thought of infants and nonhuman animals is just not as sophisticated as the linguistic, propositional thought of adult humans. Thus, I do not consider it a disadvantage of my view that thinking grounded in iconic representations is limited in both its sophistication and scope.

A final caveat regards other accounts of cognition. I do not take iconic representations to be the only possible account of cognition. Some theorists have argued that iconic representations involve descriptions that, in a sense, tell us what the image represents. Indeed, several theorists have argued that something like a LOT underlies thinking in iconic representations (Pylyshyn 1981; Tye 1991). Another possibility is of a mixed representational system consisting of both iconic and noniconic representations (Camp 2009). Thus, this account of iconic representations need not be a competitor to the LOT account. Nor does this account need to be in competition with connectionist accounts. It could very well be the case that iconic representations are realized in connectionist networks or dynamical systems. Lastly, though I find some features of the iconic representation account compelling, I am not committed to the truth of this account. The purpose of this section is to show that, contrary to Hutto's claims, nonsententialist accounts are available.

Now I turn to my argument that iconic representations satisfy Hutto's four criteria of contentful, representational mental states. First, iconic representations can be intentional. Mental iconic representations can represent things under certain descriptions or under particular modes of presentation. For example, suppose I have a mental image of a meadow from a particular vantage point. I encounter this 
same meadow but from a different vantage point. I regard these, or they are somehow tagged as mental images of different meadows, but in fact they represent the same meadow from different angles. Iconic representations can also pick out things that do not exist. Suppose I have a mental image of some landmark, which unknown to me has been completely destroyed by an ecological disaster. The landmark does not exist, yet I still have a mental image of it. Thus, iconic representations satisfy two criteria of intentionality. ${ }^{10}$

Second, iconic representations can intrinsically represent that which they are about. That is, iconic representations can have nonderived content. Consider the difference between an actual, tangible map and a mental map. The former represents, for example, the geography of a meadow only as a matter of convention. The same item could, on another interpretation, represent the economy of China. The tangible map does not intrinsically represent the meadow. Mental maps, however, do intrinsically represent. A mental map of the meadow is imagistic, but it is not its imagistic similarity to the meadow that makes it a representation of the meadow. One's mental map of the meadow may not resemble the meadow very much at all. It could in fact more closely resemble another location that the organism has never seen, and yet it is a representation of the meadow and not the other location. It is the fact that in having the iconic representation, it is tagged as a representation of the meadow. It is an essential feature of the mental map that it is a representation of the meadow. Unlike tangible, external pictures, maps, and models, mental iconic representations do not require an independent observer to interpret and assign meaning to the representations. Thus, mental images, maps, and models do intrinsically represent.

Hutto's third requirement on contentful, representational thought is that it be a part of a representational system that allows for recombination and creativity. This requirement is, in other words, the productivity and systematicity constraint argued for by Fodor and Pylyshyn (1988). A system of iconic representations is capable of a kind of productivity and systematicity. By combining iconic representations, it is possible to produce, in principle, indefinitely many distinct iconic representations. Certainly there are complex representations that this system could not produce by recombining iconic representations, but this is not problematic because the requirement is not that the representational system be able to produce any particular complex representation. (If that were the productivity requirement then even adult human thought would fail to meet it.) Thus, iconic representations satisfy the productivity criterion.

Systematicity is trickier, but once we appropriately understand what systematicity requires it is clear that some nonverbal organisms satisfy this requirement. The generality constraint is a constraint on genuine, conceptual thinking that requires that states with conceptual content be systematic (Evans 1982). It says that genuine thinkers must be capable of entertaining all syntactically permissible combinations of any concepts that they possess. Thus, if you can think that $a$ is $F$ and $b$ is $G$, then you can think that $a$ is $G$ and $b$ is F. Peter Carruthers (2009) convincingly 
argues that the generality constraint admits of both a strong and a weak version, and only the weak version, which many nonhuman animals satisfy, is warranted. The strong version holds that, "If a creature possesses the concepts $F$ and $a$ (and is capable of thinking $\mathrm{Fa}$ ) then for all (or almost all) other concepts $\mathrm{G}$ and $b$ that the creature could possess, it is metaphysically possible for the creature to think $G a$, and in the same sense possible for it to think $F b$ (Carruthers 2009, 98). The weak version holds that, "If a creature possesses the concepts $F$ and $a$ (and is capable of thinking $\mathrm{Fa}$ ) then for some other concepts $G$ and $b$ that the creature could possess, it is metaphysically possible for the creature to think $G a$, and in the same sense possible for it to think $F b^{\prime \prime}(2009,99)$. Carruthers argues that the strong version of the generality constraint is too strong, for even adult humans do not meet it. We cannot interpret syntactically well-formed thoughts like Julius Caesar is a prime number or Green ideas sleep furiously. That should lead us to reconsider the strength of the generality constraint and what satisfying the constraint is meant to show.

The generality constraint is motivated by the idea that contentful, representational thoughts must be compositionally structured. The components of thought are concepts. And for something to be a genuine concept, it must be capable of combining with at least some other concepts to create distinct thoughts. If it were impossible for the concept $F$ in $F a$ to combine with any other concept that the organism possesses, then either $F$ or $a$ or both are not really concepts; i.e., distinct isolable parts of the larger representational state (Carruthers 2009, 94-98). So what we need to show is that it is not impossible for components to recombine with other components. We do not need the strong generality constraint to show this. Adult humans most closely approximate the ideal of the strong generality constraint. But satisfying that ideal should not be the minimum standard for contentful, representational thought. To get the conclusion that organisms are capable of a certain range of contentful, representational thoughts, which is what I aim to establish with regard to nonverbal organisms, they need to satisfy only the weak generality constraint.

The behavior of nonverbal organisms satisfies the weak generality constraint. Consider the honeybees' ability to navigate by the azimuth (the compass direction of the sun). In addition to their ability to navigate by landscape cues, the mechanism that tracks the azimuth allows the bees to constantly update their location with respect to the hive and nectar. Together, these capacities allow the bees to represent the hive-to-nectar direction as well as the nectar-to-hive direction. This applies to any location to which the bees navigate, even when they navigate to novel locations, and even when they are displaced and released into unfamiliar areas (Tetzlaff and Rey 2009, 80-81). In the latter case, the original vector to a goal destination and expected landmarks along that vector conflict with the bees' current perceptual information. The honeybees use the azimuth and landmarks to recalculate and navigate a novel route back to their goal destination. These experiments show that the components of honeybees' thought-iconic representations of landmarks, the hive, sources of nectar, the solar azimuth — can be recombined in novel ways to navigate to their goal destination. The flexible use of information in serv- 
ice of different goals shows that the honeybees' cognitive system exhibits systematicity. ${ }^{11}$ This shows that nonverbal organisms' cognitive systems can exhibit productivity and systematicity, which is the third of Hutto's four requirements for contentful, representational thought.

Before moving on to the fourth criterion, I want to preempt a fairly obvious yet misguided response. One might argue that the representational system of honeybees (and other nonverbal animals) is not in fact systematic because not all of their behavior demonstrates the ability to flexibly recombine distinct representations. Pointing to some particularly rigid behaviors, one might argue that these behaviors demonstrate that such organisms are not thinking organisms. The assumption implicit in this response is that all of an organism's behavior must be guided by genuine (i.e., concept-involving, intentional, productive, systematic, logical, etc.) thought if any of it is. This assumption is surely false, for not all of adult humans' behavior is guided by genuine thought (Carruthers 2009, 91). Since we do not hold even the most sophisticated thinking creatures to this standard, it is illegitimate to employ it as a standard for less sophisticated thinking creatures. My aim in this section is to dismiss EC's argument that nonverbal intersubjective practices could not involve mindreading because they do not meet the criteria for contentful, representational cognition. Achieving this aim does not require showing that nonverbal cognition is as sophisticated as_-or more sophisticated than!adult human cognition.

The fourth criterion for genuine, contentful mental representation is that a representational system support truth-evaluable logical inferences. Iconic representations can support a limited range of logical inferences. First, we need to establish that iconic representations have a structure that is capable of supporting logical inference. Using a correspondence theory of truth, we can get a notion of true and false representations. An iconic representation is true when and insofar as it corresponds to reality and false when and insofar as it fails to correspond to reality. For example, a mental map represents the hive in a certain location relative to the nectar. The representation of the landmark as being in a particular location is true insofar as it accurately represents the actual relative location of the hive. This example also shows that mental maps are structured representations. A honeybee's mental map contains symbols (e.g., the hive, a source of nectar, and a landmark) whose content are ascribed properties (e.g., being a certain distance and direction from the hive), ${ }^{12}$ which are updated based on perception (e.g., as the solar azimuth changes), and flexibly employed in navigation (e.g., displaced honeybees use both landmarks and the mechanism that tracks the azimuth to navigate novel routes from unfamiliar territory back to their original destination). Honeybees' flexible use of spatial information and memory in the service of a multitude of different goals is key to showing that mental maps can be symbolically structured (Carruthers 2009).

The question now is whether iconic representations, like the honeybees' symbolically structured mental maps, can support logical inferences. Clearly, iconic representations cannot play the exact role that propositions play in sentential logic. It 
is unlikely that iconic representations can express De Morgan's Law (NOT (P OR $\mathrm{Q})=($ NOT P) AND (NOT Q)). But we can articulate protological roles for iconic representations. For instance, we can acquire a notion of conjunction with the combination of mental images. We can also obtain a notion of negation. It would not be a fully abstract concept of negation like the negation used in logic and natural language. If, for example, the idea of a cat is represented by a cat prototype or exemplar, then the idea of a non-cat is a combination of all the images that do not correspond to the cat prototype. One may even be able to articulate a protological disjunctive syllogism (P OR Q; NOT P; therefore Q) (Bermúdez 2003, 140-49; Prinz 2002). ${ }^{13}$ These protological concepts are not as sophisticated as our logical concepts. Given that the explanandum here is nonhuman animals' nonverbal thought and infants' preverbal thought, this is an acceptable consequence.

My discussion in this section shows that representational capacities do not require sentence-like structures. Iconic representations, which do not depend on natural language, satisfy Hutto's four necessary and jointly sufficient conditions for contentful, representational when these conditions are appropriately understood. This undermines EC's claim that genuinely contentful, representational thought requires natural language. Establishing that nonverbal organisms can have contentful, representational thought refutes the claim that primary and secondary intersubjectivity must be nonmentalistic, and so cannot involve mindreading.

\subsection{AGAINST THE NATURAL LANGUAGE REQUIREMENT}

The EC argument against the LOT hypothesis is that no theory of content could explain how mentalese sentences get their meaning. Following Kathleen Akins, Hutto considers and rejects naturalistic theories of content because they rest on a wrong account of representation and the faulty containment metaphor; i.e., the idea that signs have content on their own, they "say how things stand," "naturally indicate" or "refer to" other worldly items (Akins 2003; Hutto 2008, 48). If this were the case, "the world would be everywhere silently referring or marking truths about itself (a strange metaphysics indeed)" (Hutto 2008, 49). In contrast to theories that succumb to the containment metaphor, Hutto states that natural signs or signals represent something only if they are used as entities that stand for something else. Having rejected the standard kind of theory of content associated with LOT, Hutto concludes, "[S]ince I know of no other viable way of accounting for subpersonal content-bearing mental states, I am inclined to deny the existence of nonverbal beliefs. This leads me to conclude that 'believing propositions', in the sense of apprehending them under intensional modes of presentation, is not the fundamental way in which we or other animals relate to worldly offerings" (Hutto 2008, 109).

Hutto says we should reject naturalistic theories of content because they are committed to a mistaken account of representation and the allegedly dubious containment metaphor. I think the containment metaphor discussion is a red herring. For one could perfectly consistently hold that things in the world carry informa- 
tion about other things, but in order for thing $(\mathrm{P})$ to represent other thing $(\mathrm{Q}), \mathrm{P}$ must additionally stand in for Q. Everyone, after all, thinks that representation requires more than mere informational covariance. ${ }^{14}$ Thus, the real dispute here is about representation—as it often is with EC (Gibson 1986; Noë 2006).

Hutto offers no reason to reject others' accounts of representation, and he offers no reason to accept his preferred account of representation. After explicating Dretkse's teleological causal-informational theory (which I described above), Hutto claims, "But this is a mistake. Natural signs (or signals) only serve to 'represent' anything at all if they are used in a very particular way — that is, as 'entities which stand for something else"' $(2008,47)$. Hutto simply asserts that this is the correct account of representation and cites Rick Grush (2003).

There are many accounts of representation in the cognitive sciences. William Ramsey (2007) discusses at least six distinct accounts of representation that are employed in psychology, neuroscience, and philosophy of mind. Hutto's preferred account is but one among several legitimate accounts of representation. It is plausible that each of these accounts is appropriate for some area of cognitive science and inappropriate for other areas. Hutto needs to show that the accounts of representation on which theorists like Dretske rely are inappropriate for understanding social cognition.

Not only does Hutto not show this, his own account of basic embodied responding is remarkably similar to Dretske's characterization of contentful representations. ${ }^{15}$ Hutto says, "Organisms of all kinds are informationally sensitive to select worldly offerings-sometimes in complex ways. These built-in sensitivities permit them to identify and track certain environmental particulars of importance (to them); detection of these particulars initiates responses that enable the coordination of successful actions in certain environments. This package will have been selectively forged in the service of meeting the end-directed needs of specific creatures" $(2008,50)$. For example, humans are informationally sensitive to certain facial configurations. These facial configurations and our sensitivity to them have the biological proper function of enabling successful coordination among people. This account does not significantly differ from Dretkse's account, which would describe facial configurations as having the function of indicating certain emotions.

In arguing against LOT, Hutto aims to establish that there is no plausible account of nonverbal propositional thought. As I have shown in this section, the case against LOT rests on the mere assertion that the stand-in account of representation is the only legitimate notion of representation. Hutto does not provide any evidence that other kinds of representation are inappropriate for this domain. And in fact his own description of nonverbal organisms' behavior differs only terminologically from Dretske's account of representation. Hutto's argument against LOT rests on a rejection of naturalistic theories of content; however, he has failed to refute any naturalistic theory of content. Thus, the argument against LOT is unsuccessful. EC fails to establish that nonverbal organisms could not have propositional thought. 


\subsection{THE INADEQUACY OF EC'S ACCOUNT}

In the previous sections I argued that EC fails to refute LOT and nonsententialist accounts that ascribe contentful, representational thought to nonverbal organisms. In this section I shall argue that EC's nonmentalistic account offers inadequate explanations of some behavior. I shall focus on relatively simple behavior of nonverbal organisms and argue that even for this relatively simple behavior EC's nonmentalistic account is inadequate. Some nonverbal behavior is best understood in terms of contentful, representational thought. If the EC account is too deflationary to explain simple nonverbal behavior, then it is even more woefully inadequate as an account of human behavior. For the social cognition of some nonhuman animals and preverbal children is much more sophisticated than the examples I use in this section.

I shall use honeybee behavior as my example. Honeybees integrate and constantly update highly specific information about distance, time, direction, and landmarks in order to navigate to and from the hive. I think the best explanation is that honeybees represent and protologically reason about the location of the hive. Hutto explains the waggle dance in terms of evolutionarily selected indexical guides that directly guide the dancing and observing honeybees' behavior. He argues that for the waggle dance to guide behavior successfully neither the dancing nor the observing honeybees need represent anything. The honeybees only need to respond appropriately to certain cues in order to act successfully.

Simply focusing on the waggle dance may make such a description seem adequate, but when one also incorporates how honeybees navigate, the deflationary description begins to look far too deflationary. Honeybees' behavior is flexible. They rely on memory of landmarks. It seems that they distinctly represent things in their environment. They can navigate novel routes to their destination when they are relocated to unfamiliar areas and receive perceptual information that conflicts with the expected perceptual information. And their behavior meets all of the criteria set forth for contentful mental representations. If honeybee behavior were simply a matter of evolutionarily selected indexical guides; that is, if honeybees behaved according only to hard-wired behavioral scripts, we would expect all of their behavior to be extremely rigid. But their behavior is not rigid. Honeybees' navigational skills are incredibly flexible, as demonstrated by their ability to navigate novel routes from unfamiliar areas back to the hive. It is precisely this behavioral flexibility that leads theorists to describe honeybee behavior in representational terms. The flexibility of honeybees' navigation strongly suggests that honeybees are capable of symbolic thought; i.e., thinking that involves symbols that represent things in the world. It is difficult to see how the deflationary account could plausibly explain the impressive flexibility of honeybee behavior with only evolved hard-wired behavioral scripts.

Throughout this discussion I have used honeybee behavior as my primary example. I have done this only for the sake of clarity and convenience. We could just 
as easily use examples from studies on primates, birds (western scrub jays are particularly impressive), dogs, or human infants. ${ }^{16}$ All of these organisms lack facility with natural language and yet display flexible behavior that is best described as guided by contentful representational mental states.

The discussion so far shows three things. First, the EC argument against contentful, representational nonverbal thought fails. It does not refute LOT or nonsententialist accounts of nonverbal thought. Second, we can give a plausible account of nonverbal reasoning in terms of iconic representations. ${ }^{17}$ Third, EC's own account of nonverbal behavior is ill equipped to explain the empirically well-established flexibility of even nonverbal organisms' behavior. The plausibility of mentalistic accounts of nonverbal behavior-i.e., accounts that regard some nonverbal behavior as guided by contentful, representational mental states, and the corresponding implausibility of nonmentalistic accounts - indicates that EC fails to present a compelling alternative to the standard account.

\subsection{REJECTING THE EVIDENCE FOR EC}

The final topic of this section involves the evidence corralled for the idea that contentful, representational thought depends on natural language. Hutto takes as evidence for this claim the fact that congenitally deaf children raised in nonsigning households perform poorly on the standard false-belief task until they learn sign language and the fact that development of language enhances performance on mindreading tasks $(2008,131-42)$.

The first thing to note is that this evidence is meant to work in conjunction with the previous arguments because otherwise it could not establish that both having and attributing mental states depends on language mastery. On its own, the evidence that oral deaf children perform poorly on the standard false-belief task bears only on the issue of attributing mental states. Since at this point we have dismissed the arguments against the possibility of nonverbal creatures having contentful, representational mental states, the question now is whether this evidence tells against the claim that nonverbal creatures can attribute mental states. I do not think it does.

The standard false-belief task relies on an elicited response paradigm according to which an experimenter asks the subject a question, the subject must correctly interpret the question, figure out an answer, and convey that answer to the experimenter. This process relies heavily on language and executive function; e.g., memory and response inhibition. There are convincing arguments that this task is too difficult even for normally developing children, let alone those who are severely limited in their ability to communicate with others. The fact that oral deaf children perform poorly on this task could show that they have serious mindreading deficits that are due to their lack of language. Or these results could show that the elicited response paradigm is simply too linguistically challenging for oral deaf children. At multiple stages in the task, subjects are required to interpret and produce appropriate linguistic responses. If their poor performance on the standard false-belief task 
stemmed primarily from difficulty with the linguistic task demands, then it would be utterly unsurprising that their performance improved upon mastering sign language. At that point, the children could easily interpret and communicate appropriate linguistic responses. ${ }^{18}$ If this interpretation is right, then these results are not evidence that attributing mental states depends on the mastery of natural language. Since the standard false-belief task cannot test between mindreading competence and task performance, it is poor evidence for the claim that attributing mental states depends on mastery of natural language.

The other sources of evidence are that performance on the standard false-belief task improves at roughly the same time that children's linguistic abilities begin to mature and that development of language enhances performance on mindreading tasks. As just noted, the standard false-belief task tests as much for executive controls (e.g., memory and response-inhibition) and linguistic abilities as it does for facility with mental state attribution. Thus, the fact that success on the standard false-belief task coincides with mastery of natural language is unilluminating in this discussion. In fact, it is for this very reason that most philosophers and psychologists no longer regard success on the standard false belief task as a measuring stick of mindreading abilities (Bloom and German 2000; Goldman 2006, 69-78; Nichols and Stich 2003, 74). Furthermore, the claim that mental state attribution and mastery of language occur at the same time ignores much of the current research in developmental psychology, which aims to establish that infants have rudimentary mindreading abilities. Some theorists argue that even seven-month-olds are sensitive to others' mental states (Kovacs 2009). Developmental psychologists and philosophers have long abandoned the idea that success on the standard false-belief task at age four is necessary and sufficient for sophisticated folk psychological competence, so it is strange that EC remains wedded to this abandoned developmental timeline.

In any case, the fact that the onset of language coincides with success on the standard false-belief task has several possible explanations. Mastery of language and success on the standard false-belief task may have a common cause-e.g., development of the prefrontal cortex-which enables cognitive control over attention, response inhibition, and decision making. If this were the case, the common cause could (partially) explain why only humans develop natural language and sophisticated mindreading. Another (not incompatible) explanation is that language and mindreading co-develop. Or it may be the case that maturing mindreading abilities make possible the development of language. In fact, it seems that we need some mindreading abilities to learn language. To learn the meanings of words and to form various concepts, the child needs to grasp the speaker's referential intention. All three of these explanations would account for the data and all three are incompatible with the EC claim that mindreading depends on language mastery. The empirical data Hutto cites do not discriminate among these distinct, competing hypotheses, and so they are poor evidence for the EC account of social cognition. 


\section{ASSESSMENT}

In this paper I considered an argument for embodied social cognition. The argument rests on three premises. First, only a sentential medium could support contentful, representational thought. Second, natural language is the only legitimate source of sententially based thought. Thus, preverbal and nonverbal creatures cannot have, let alone attribute to others, contentful, representational thoughts. Third, the best explanation of preverbal and nonverbal cognition is in terms of nonmentalistic intentional attitudes. Together, these premises are meant to establish that mindreading is not available as a means of understanding others until relatively late in human cognitive development.

This argument aims to undermine the developmental timeline of mindreading accounts, which hold that human infants and perhaps some nonhuman animals engage in mindreading in at least a rudimentary form. If successful, the argument would also put pressure on the idea that mindreading is our fundamental mode of understanding others. According to embodied social cognition, nonmentalistic embodied practices are in place prior to the ability to mindread, they underlie the higher-level, more specialized, rare cognitive skills involved in mindreading, and they are the primary way we continue to understand others.

The argument for embodied social cognition is unsuccessful, however. All three premises motivating the deflationary account of social cognition fail. In particular, EC fails to refute LOT and nonsententialistic accounts of thought and cannot plausibly explain the flexibility of the behavior of even relatively simple organisms. Moreover, the evidence cited in favor of EC does not in fact support the EC account. As a result, the case for embodied social cognition fails.

\section{NOTES}

1. Most EC theorists do not deny that we can and sometimes do think about others' thoughts. They deny that this is a fundamental, widespread part of social cognition. Some EC theorists go even further than this claim, arguing that we never attribute mental states to others. Ratcliffe (2005) for example, is an eliminativist about folk psychology. I shall not consider eliminativist varieties of EC here.

2. For a critical appraisal of these arguments, see Spaulding (2010).

3. See also Davidson (2001) and Wittgenstein (1953) and their intellectual descendants for a similar view.

4. Fodor's asymmetric dependence account differs from Dretske's teleological account in its specification of the further requirements on causal-informational relations.

5. Akins (2003) makes a similar argument.

6. Hutto's account draws heavily from Millikan's account of biosemantics (2005). Millikan, however, defends a notion of representational content in terms of biological proper functions. Hutto regards representational content as an unnecessary and unhelpful part of the account and so calls his account biosemiotics rather than biosemantics $(2008,50-59)$. 
7. For the EC theorist to get the conclusion that even as adults our ordinary social engagements are nonmentalistic, one would additionally have to argue that adults' ordinary social engagements do not consist in thinking in natural language and that our ability to think in natural language does not fundamentally alter our basic intersubjectivity.

8. In what follows, for the sake of convenience I will treat nonverbal and preverbal thought as if they are cognitively similar. My argument is a response to the claim that nonverbal and preverbal behavior are alike in being nonmentalistic. I shall argue that nonverbal and preverbal behavior are not necessarily nonmentalistic, but my account is not committed to the idea that nonverbal and preverbal organisms are cognitively identical. After all, preverbal infants go on to develop sophisticated cognitive abilities that nonverbal nonhuman animals, even when they are reared in human environments, do not.

9. Mental images are representations akin to perceptual experiences. They are not literally pictures in the brain. A mental image is similar to a mental map, which I understand to be "a record in the central nervous system of macroscopic geometric relations among surfaces in the environment used to plan movements through the environment" (Gallistel 1990, 103). Mental models, which are also similar to mental maps, are representations of one's interaction with the environment, other organisms, and artifacts in the environment. Mental models are error-prone, oversimplified, constantly updated representations of reality that one uses to understand phenomena (Johnson-Laird 1983).

10. See Millikan (2005) and Carruthers (2006) for arguments that honeybees' mental maps are intentional.

11. See Tetzlaff and Rey (2009) and Carruthers (2009) for an extended defense of this idea.

12. I take these to be de re rather than de dicto property ascriptions. In other words, the honeybees regard what we call the hive as being what we measure as 10 meters away. This does not entail that honeybees have the concept of a meter, the number 10, etc.

13. The case of Chrysippus's dog illustrates this kind of reasoning. Chrysippus's dog, "when, on arriving at a spot where three ways meet ..., after smelling at the two roads by which the quarry did not pass, he rushes off at once by the third without stopping to smell. For, says the old writer, the dog implicitly reasons thus: 'The animal went either by this road, or by that, or by the other: but it did not go by this or that, therefore he went the other way'" (quoted from Rescorla 2009, 53). Historically, there have been two main sides in the debate about the dog's reasoning powers: either the dog has fairly sophisticated logical reasoning abilities or the dog has no logical reasoning abilities and the appearance of reasoning can be explained away by behavioral rules. My goal in advancing a protological account of iconic representations is to articulate a middle ground between these two positions.

14. For what it is worth, the arguments in the discussion of the containment metaphor are unconvincing anyway. Hutto offers only two considerations against the idea that things naturally indicate: the strange metaphysics remark (which if it is an argument is a patently question-begging argument) and the claim that the containment metaphor leads theorists to mistakenly believe that signals must be decoded and understood by organisms in order for such signals to successfully guide action. This latter claim is allegedly wrong because accounting for nonverbal organisms' successful behavior only requires purely physical, behavioral explanation. Hutto's mistaken assumption is that it is legitimate to impute computational or representational cognition only when such an explanation is required. But we are never required to describe a system in such terms. Purely physical, behavioral explanations are always available. The question is whether it is useful to describe behavior in computational or representational terms, and Hutto does not answer that question.

15. As noted above, his account is also very similar to Millikan's account of biosemantics.

16. See, for example, Tomasello and Call (1997), Dally et al. (2006), Clatyon et al. (2007), and Carruthers (2006).

17. Such an account is not necessarily incompatible with LOT or with another kind of mixed-representation system.

18. This is not to deny that language is relevant to mindreading. Surely being walled off from linguistic interactions has a deleterious effect on social interactions and maturation of social cognition. There are some interactions in which these children simply cannot engage. Mastering natural lan- 
guage will enhance cognitive abilities in general, and that includes social cognition. These facts do not, however, establish that attributing mental states depends on the mastery of natural language. Thanks to Robert Thompson for helpful discussion on this point.

\section{REFERENCES}

Akins, K. 2003. "Of Sensory Systems and the 'Aboutness' of Mental States." In Philosophy and the Neurosciences: A Reader. Ed. W. Bechtel, P. Mandik, J. Mundale, and S. Stufflebeam. Oxford: Blackwell. 369-94.

Bermúdez, J. L. 2003. Thinking without Words, Vol. 11. Oxford: Oxford University Press.

Bloom, P., and T. P. German. 2000. "Two Reasons to Abandon the False Belief Task as a Test of Theory of Mind." Cognition 77(1): B25-B31.

Camp, E. 2009. "A Language of Baboon Thought?" In The Philosophy of Animal Minds. Ed. R. W. Lurz. Cambridge: Cambridge University Press.

Carruthers, P. 2006. The Architecture of the Mind. New York: Oxford University Press.

Carruthers, P. 2009. "Invertebrate Concepts Confront the Generality Constraint (and Win)." The Philosophy of Animal Minds. Cambridge: Cambridge University Press.

Clayton, N. S., J. M. Dally, and N. J. Emery. 2007. "Social Cognition by Food-Caching Corvids: The Western Scrub-jay as a Natural Psychologist." Philosophical Transactions of the Royal Society B: Biological Sciences 362(1480): 507-22.

Dally, J., N. Emery, and N. Clayton. 2006. "Food-Caching Western Scrub-jays Keep Track of Who Was Watching When." Science 312(5780): 1662.

Davidson, D. 2001. Essays on Actions and Events. 2nd ed. Oxford: Clarendon.

de Bruin, L. C. 2008. "A New Story about Folk Psychology." Philosophical Explorations 11(3): 263-71.

Dretske, F. 1988. Explaining Behavior: Reasons in a World of Causes. Cambridge, MA: MIT Press.

Dyer, F. 2002. "The Biology of the Dance Language." Annual Review of Entomology 47(1): 917-49.

Evans, G. 1982. Varieties of Reference. Oxford: Oxford University Press.

Fodor, J. A. 1975. The Language of Thought. Cambridge, MA: Harvard University Press.

Fodor, J. A., and Z. W. Pylyshyn. 1988. "Connectionism and Cognitive Architecture." Cognition 28: 3-71.

Gallagher, S. 2005. How the Body Shapes the Mind. New York: Oxford University Press.

Gallagher, S. 2008. "Inference or Interaction: Social Cognition without Precursors." Philosophical Explorations 11(3): 163-74.

Gallagher, S., and D. D. Hutto. 2008. "Understanding Others through Primary Interaction and Narrative Practice." In The Shared Mind: Perspectives on Intersubjectivity. Ed. Sinha, Itkonen, Zlatev, and Racine. Amsterdam: John Benjamins.

Gallistel, C. 1990. The Organization of Learning. Cambridge, MA: MIT Press.

Gibson, J. J. 1986. The Ecological Approach to Visual Perception. London: Lawrence Erlbaum.

Goldman, A. I. 2006. Simulating Minds: The Philosophy, Psychology, and Neuroscience of Mindreading (Philosophy of Mind). New York: Oxford University Press.

Grush, R. 2003. "The Architecture of Representation." In Philosophy and the Neurosciences: A Reader. Ed. W. Bechtel, P. Mandik, J. Mundale, and S. Stufflebeam. Oxford: Blackwell. 349-68.

Hutto, D. D. 2008. Folk Psychological Narratives: The Sociocultural Basis of Understanding Reasons. Cambridge, MA: MIT Press.

Johnson-Laird, P. 1983. Mental Models: Towards a Cognitive Science of Language, Inference and Consciousness. Cambridge, MA: Harvard University Press.

Kovacs, A. 2009. "Early Bilingualism Enhances Mechanisms of False-Belief Reasoning." Developmental Science 12(1): 48-54.

Millikan, R. G. 2005. “The Father, the Son, and the Daughter: Sellars, Brandom, and Millikan.” Pragmatics and Cognition 13(1): 59-71.

Nichols, S., and S. Stich. 2003. Mindreading: An Integrated Account of Pretence, Self-Awareness, and Understanding Other Minds. Oxford: Oxford University Press.

Noë, A. 2006. Action in Perception. Cambridge, MA: MIT Press.

Peterson, C. C., and M. Siegal. 2000. "Insights into Theory of Mind from Deafness and Autism." Mind and Language 15(1): 123-45. 
Prinz, J. J. 2002. Furnishing the Mind: Concepts and Their Perceptual Basis. Cambridge, MA: MIT Press.

Pylyshyn, Z. 1981. “The Imagery Debate: Analogue Media Versus Tacit Knowledge.” Psychological Review 88(1): 16-45.

Ramsey, W. M. 2007. Representation Reconsidered. Cambridge: Cambridge University Press.

Ratcliffe, M. 2005. "Folk Psychology and the Biological Basis of Intersubjectivity." Royal Institute of Philosophy Supplements 80(56): 211-33.

Rescorla, M. 2009. “Chrysippus' Dog as a Case Study in Non-linguistic Cognition.” In The Philosophy of Animal Minds. Ed. R. W. Lurz. Cambridge: Cambridge University Press.

Riley, J., U. Greggers, A. Smith, D. Reynolds, and R. Menzel. 2005. “The Flight Paths of Honeybees Recruited by the Waggle Dance." Nature 435(7039): 205-7.

Spaulding, S. 2010. "Embodied Cognition and Mindreading." Mind \& Language 25(1): 119-40.

Tetzlaff, M., and G. Rey. 2009. "Systematicity and Intentional Realism in Honeybee Navigation." The Philosophy of Animal Minds, 72-88.

Tomasello, M., and J. Call. 1997. Primate Cognition. Oxford: Oxford University Press.

Tye, M. 1991. The Imagery Debate. Cambridge, MA: MIT Press.

Von Frisch, K. 1967. The Dance Language and Orientation of Bees. Cambridge, MA: Belknap Press of Harvard University Press.

Wittgenstein, L. 1953. Philosophical Investigations. Trans. G. E. M. Anscombe. London: Blackwell. 